\title{
SINTOMAS DE ESTRESSE EM FAMILIARES DE PACIENTES ADULTOS EM TERAPIA INTENSIVA
}

\section{Tatiele Galli Zanetti}

Enfermeira, Especialista em Unidade de Terapia Intensiva, Brasil.

\section{Sandra Leontina Graube}

Enfermeira, Mestranda pelo Programa de Pós-graduação Stricto sensu em Atenção Integral à Saúde na Universidade Regional do Noroeste do Estado do Rio Grande do Sul (UNIJUI), Ijuí (RS) Brasil.

E-mail: graubesandra@yahoo.com.br

\section{Cátia Cristiane Matte Dezordi}

Enfermeira. Mestranda pelo Programa de Pós-graduação Stricto sensu em Atenção Integral à Saúde na Universidade Regional do Noroeste do Estado do Rio Grande do Sul (UNIJUI), Ijuí (RS) Brasil.

\section{Vivian Lemes Lobo Bittencourt}

Enfermeira. Mestre, Docente na Universidade Regional Integrada do Alto Uruguai e das Missões (URI), Santo Ângelo (RS), Brasil.

\section{Roberta Cattaneo Horn}

Farmacêutica, Doutora, Docente Adjunta da Universidade de Cruz Alta (UNICRUZ), Cruz Alta (RS), Brasil.

\section{Eniva Miladi Fernandes Stumm}

Enfermeira. Doutora, Docente Adjunto I na Universidade Regional do Noroeste do Estado do Rio Grande do Sul (UNIJUÍ), Ijuí (RS) Brasil.
RESUMO: Correlacionar sintomas físicos e psicológicos das fases de estresse identificadas em familiares de pacientes internados em Unidade de Terapia Intensiva Adulto. Estudo quantitativo, transversal, analítico, realizado em um hospital geral do Rio Grande do Sul. A amostra foi composta por 22 familiares e para coleta de dados foi utilizado formulário sociodemográfico e Inventário de Sintomas de Estresse. Constatou-se que 45,5\% dos participantes da pesquisa encontravam-se na fase final e 40,9\% na intermediária. Houve correlação estatisticamente significante entre número de sintomas fisiológicos e psicológicos presentes nas diferentes fases. $\mathrm{O}$ reconhecimento de sintomas de exaustão em familiares de pacientes em terapia intensiva pelo enfermeiro é importante aliado às estratégias de enfrentamento adequadas, que incluem acolhimento, instrumentalização, orientação e apoio aos familiares neste período.

PALAVRAS-CHAVE: Unidade de terapia intensiva; Família; Estresse psicológico; Enfermagem.

\section{STRESS SYMPTOMS IN RELATIVES OF ADULT PATIENTS UNDERGOING INTENSIVE THERAPY}

\begin{abstract}
Current qualitative, transversal and analytic study corelates physical and psychological symptoms of stress phases identified in relatives of hospitalized patients in ITUs in a general hospital in the state of Rio Grande do Sul, Brazil. The samples comprised 22 relatives. A socio-demographic form and the Stress Symptoms Inventory were employed for data collection. Data show that $45.5 \%$ of participants in research were in the final phase and $40.9 \%$ in the intermediate phase. There was a statistically significant co-relationship between the number of physiological and psychological symptoms in the different phases. Acknowledgement of exhaustion in relatives of patients in ITUs by nurses is important, coupled to adequate coping strategies. These include welcome, instrumentalization, guidance and help to relatives during the period.
\end{abstract}

KEY WORDS: Intensive Care Unit; Family; Psychological stress; Nursing.

\section{INTRODUÇÃO}

A Unidade de Terapia Intensiva (UTI) caracteriza-se por ser um local de alta complexidade tecnológica, com a finalidade de reestabelecer a saúde de pacientes de alto risco, com equipe 
multiprofissional, materiais e equipamentos para o monitoramento constante, de maneira a proporcionar agilidade e eficácia na assistência (SIMONI; SILVA, 2012). A referida unidade é avaliada como um ambiente estressante pelos pacientes, familiares e equipe de saúde (NASCIMENTO; GULINI; MINUZZI et al., 2016).

A internação em UTI, associada à gravidade clínica do paciente, falta de conhecimento, presença de tubos e sondas, impossibilidade de comunicação e limitação de movimentos são situações descritas como estressores e desencadeadores de ansiedade, aliado ao fato de o paciente estar impossibilitado de tomar decisões e essas responsabilidades recaírem sobre os familiares (DREFFS; STUMM; WINKELMANN et al., 2013).

A apreensão dos familiares frente à doença, que se constitui em uma ameaça à vida do paciente, provoca alterações no equilíbrio familiar que pode agravar-se no decorrer da internação em UTI. O fato de os familiares permanecerem na sala de espera e acompanharem o movimento de pacientes, profissionais e familiares dos demais internados, associado à restrição de visitas e, principalmente, de informações, contribuem para a ansiedade e os efeitos dela decorrentes (ZANETTI; STUMM; UBESSI, 2013).

Nesse contexto, a internação em UTI contribui para a ocorrência de diversos sentimentos para os pacientes e seus familiares. Esses anseios transcorrem principalmente pelo fato de a hospitalização, na maioria das vezes, ocorrer de forma súbita, o que faz com que a família tenha pouco tempo para se ajustar à situação (FERREIRA; MENDES, 2016).

Diante dessas circunstâncias, o relacionamento familiar pode vivenciar situações frustrantes o que resulta em alterações físicas ou psicológicas dos seus integrantes (MOMBELLI; COSTA; MOURA et al., 2011). Assim, os familiares de pacientes em terapia intensiva vivenciam um impacto emocional pela internação, a angústia decorrente do futuro incerto e a possibilidade iminente da morte (NASCIMENTO; GULINI; MINUZZI et al., 2016).

Considera-se que o equilíbrio da família pode ser comprometido no momento da internação diante da separação desta de um dos seus integrantes, o qual passa a ser cuidado por profissionais da saúde. Neste momento, os sentimentos da família são intensificados, nasce a impossibilidade de um convívio direto e os familiares vivenciam sintomas de angústia (FERREIRA; MENDES, 2016).

O colapso emocional prepara o organismo para enfrentar situações de risco ou emoções fortes, contudo este é considerado uma reação normal e necessária para a sobrevivência do ser humano. O que lesa o organismo é a presença deste sentimento em níveis elevados, permanentemente, de maneira a originar sintomas físicos e psicológicos no indivíduo (GOMES; CAPELLARI; PEREIRA et al., 2016).

As manifestações do estresse ocorrem em três etapas e cada uma delas compreende um conjunto de sintomas físicos e psicológicos (CAVALCANTE; SILVA; MENDONÇA et al, 2013). Suas consequências incluem, falta de ânimo, falta de envolvimento com outros indivíduos, forma como o sujeito consegue suportar situações desgastantes, principalmente pessoas que convivem com o sofrimento, dor, morte e tantos outros sentimentos e reações advindas do processo de adoecimento (SIMÕES; BIANCHI, 2016).

Quanto mais avançada a fase, maior a intensidade e a gravidade dos sintomas apresentados e maior é a probabilidade do surgimento de doenças, em especial as cardiovasculares, também associadas ao estilo de vida (WOTTRICH; ÁVILA; MACHADO et al., 2011).

O desenvolvimento do esgotamento emocional é subdividido em fase inicial, intermediária e final. Os principais sintomas na fase inicial ou alerta vão desde desconforto gástrico até diarreia passageira, extremidades frias, boca seca, insônia, taquicardia, dentre outros. Na fase intermediária ou de resistência o corpo se adapta a estes agentes e podem permanecer sintomas da fase inicial e surgir outros, tais como pensamento focalizado em um assunto, problemas de memória, sensibilidade aumentada, mal-estar e tontura (CAVALCANTE; SILVA; MENDONÇA et al., 2013). A fase final ou de exaustão é mais aguda e caracteriza-se pelo esgotamento da energia usada na fase anterior e pela exposição prolongada do indivíduo aos agentes extenuantes (BARBOSA; SANTOS; BARBOSA, 2012).

Nesse sentido, nas últimas duas fases, pode ocorrer o adoecimento, no qual os órgãos com maior vulnerabilidade genética ou adquirida apresentam 
sinais de deterioração e adoecem (CAVALCANTE; SILVA; MENDONÇA et al, 2013). Assim, diminui a concentração, afeta a produtividade no trabalho e a pessoa apresenta atitudes muitas vezes impensadas (BARBOSA; SANTOS; BARBOSA, 2012). Desta forma, o objetivo do estudo é correlacionar sintomas físicos e psicológicos das fases de estresse identificadas em familiares de pacientes internados em Unidade de Terapia Intensiva Adulto.

\section{MÉTODOLOGIA}

Trata-se de um estudo transversal, analítico com abordagem quantitativa, realizado com 22 familiares de pacientes internados em UTI Adulto de um hospital geral, porte IV, situado na região Noroeste do Rio Grande do Sul. Esta instituição de saúde conta com 243 leitos e destes 11 leitos pertencem à UTI Adulto, com uma taxa de ocupação mensal de $82,15 \%$.

Os dados foram coletados no mês de fevereiro de 2010, na sala de espera da UTI. Foram convidados a participar 30 familiares e, destes, oito recusaram. Assim, a população foi composta por 22 familiares. Os critérios de inclusão utilizados foram idade maior que 18 anos, ser ou considerar-se membro da família do paciente internado.

Para a coleta de dados foi utilizado um formulário com dados sociodemográficos dos familiares, elaborado pelas pesquisadoras e o Inventário de Sintomas de Stress ou Estresse? de Lipp (LIPP, 2000). Para a determinação de cada fase procedeu-se da seguinte maneira: F0 - Eustresse (soma menor que 5), F1 - Fase inicial ou fase de alerta (soma igual ou superior a 5), F2 - Fase intermediária ou fase de resistência (somatório igual ou maior que 3) e F3 - Fase final ou fase de exaustão (somatório maior ou igual a 8 sintomas).

Do total de sintomas referidos pelos familiares integrantes da pesquisa, foi determinado o nível de estresse em que eles se encontravam no momento da aplicação do referido instrumento. Cabe salientar que o instrumento possibilita que um mesmo sujeito seja classificado em mais de uma fase, porém, para efeito de análise, classificou-se o familiar de acordo com o nível mais alto atingido.

Após a coleta os dados foram armazenados no
Excel for Windows e, foi utilizado o software SPSS v.23 (StatisticalPackage for Social Sciences) para todas as análises estatísticas. Para verificar associações entre o número de sintomas presentes em cada fase utilizou-se o teste de correlação de Spearman.

Os aspectos éticos foram respeitados, conforme as Diretrizes e Normas Regulamentadoras de Pesquisas que Envolve Seres Humanos, conforme a resolução do Conselho Nacional de Saúde (CNS) nº. 466/2012, de forma que o estudo foi submetido e aprovado pelo Comitê de Ética em Pesquisa da Unijuí, em 18/01/2010, sob parecer consubstanciado $n^{\circ} 009 / 2010$.

\section{RESULTADOS}

A pesquisa contou com a participação de 22 familiares de pacientes internados em UTI Adulto. Do total de familiares $77,3 \%$ do sexo feminino e com média de idade de 44,36 anos, 59,1\% casados, 40,9\% solteiros, viúvos ou separados, $86,4 \%$ com filhos, $100 \%$ professam algum tipo de crença ou religião e $72,8 \%$ com ensino fundamental incompleto.

As fases de estresse em que os familiares encontravam-se no momento da coleta de dados são apresentadas na Tabela 1 .

Tabela 1. Fases de estresse dos familiares de pacientes internados na UTI adulto ( $N=22$ ). Ijuí, RS, 2010

\begin{tabular}{lcc}
\hline Fase de estresse & $\mathbf{N}$ & $\%$ \\
\hline Estresse & 2 & 9,1 \\
\hline Fase final & $\mathbf{1 0}$ & $\mathbf{4 5 , 5}$ \\
\hline Fase inicial & $\mathbf{1}$ & $\mathbf{4 , 5}$ \\
\hline Fase intermediária & 9 & 40,9 \\
\hline Total & $\mathbf{2 2}$ & $\mathbf{1 0 0}$ \\
\hline
\end{tabular}

No que se refere aos sintomas fisiológicos presentes em cada fase de estresse, evidenciam-se que existe uma correlação significativa entre todas elas, bem como o total de sintomas referidos pelos participantes da pesquisa. 
Tabela 2. Correlação de Spearman do número de sintomas fisiológicos em cada fase definida pelo "Inventário de Sintomas de Stress" de Lipp (2000). Ijuí, RS, 2010

\begin{tabular}{lcccc}
\hline & & \multicolumn{2}{c}{ Sintomas fisiológicos } \\
\cline { 2 - 5 } & Fase inicial & Fase intermediária & Fase final & Total \\
\hline Fase Inicial & 1 & $0,809^{\#}$ & $0,785^{\#}$ & $0,950^{\#}$ \\
Fase Intermediária & & 1 & $0,693^{\#}$ & $0,914^{\#}$ \\
Fase Final & & & 1 & $0,870^{\#}$ \\
\hline
\end{tabular}

\# correlação significante $(\mathrm{p}<0,01)$

Na Tabela 3 é demonstrada a correlação do número de sintomas psicológicos referidos pelos familiares dos pacientes internados na UTI, em cada uma das fases de estresse. Nesta constata-se que há uma correlação estatisticamente significativa entre os sintomas presentes nas fases intermediária e final do estresse, bem como entre o total de sintomas psicológicos apresentados pelos participantes.

Tabela 3. Correlação de Spearman do número de sintomas psicológicos em cada fase definida pelo "Inventário de Sintomas de Stress" de Lipp (2000). Ijuí, RS, 2010

\begin{tabular}{lcccc}
\hline & \multicolumn{2}{c}{ Sintomas psicológicos } \\
\cline { 2 - 5 } & Fase inicial & Fase intermediária & Fase final & Total \\
\hline Fase Inicial & 1 & $-0,265$ & $-0,174$ & $-0,086$ \\
Fase Intermediária & & 1 & $0,689^{\# \#}$ & $0,824^{\# \#}$ \\
Fase Final & & 1 & $0,961^{\# \#}$ \\
\hline
\end{tabular}

${ }^{\#}$ correlação significante $(\mathrm{p}<0,01)$

\section{DISCUSSÃO}

No presente estudo identificou-se maioria dos participantes do sexo feminino. Esse resultado demonstra que dentre os vários papéis assumidos pela mulher, está o de cuidadora, evidenciado historicamente e o mais assimilado perante a sociedade (BARBOSA; SANTOS; BARBOSA, 2012; DREFFS; STUMM; WINKELMANN et al., 2013). A representação de vários papéis entre o cuidar da pessoa hospitalizada, ocasionam prostração e sofrimento, o que acaba por exigir a superação dos desafios apresentados no seu cotidiano (FERREIRA; MENDES, 2016).

Com base nos dados obtidos nesta pesquisa observa-se que mais da metade são casados e possuíam filhos, semelhante a outro estudo que avaliou e comparou as fases de estresse e o método de enfrentamento deste processo (ZANETTI; STUMM; UBESSI, 2013).
Este resultado denota que estes acompanhantes possuíam família constituída, ou seja, além daquele ente hospitalizado, existiam mais pessoas que necessitavam de sua atenção. No início toda a família se sensibiliza, tanto com o paciente quanto com o cuidador, porém com o passar do tempo eles se acostumam e a pessoa que assumiu a responsabilidade do cuidado se sente sozinha no processo do cuidar e renuncia, muitas vezes, de sua própria vida em prol do cuidado do outro (BAPTISTA; BEUTER; PERLINI et al., 2012).

No que se refere à escolaridade dos pesquisados, evidenciou-se que é baixa, visto que a maioria havia cursado, parcialmente, o ensino fundamental. Dado similar emergiu em pesquisa realizada com o intuito de avaliar os níveis de estresse em pacientes renais crônicos submetidos a tratamento hemodialítico (WOTTRICH; ÁVILA; MACHADO et al., 2011). O baixo nível de escolaridade pode prejudicar o entendimento da real 
situação vivida, e, dessa forma, contribuir para aumentar o esgotamento emocional vivenciado pelo fato de ter um familiar internado em UTI (ABREU; SENA; OLIVEIRA et al., 2013).

A idade dos participantes desta pesquisa variou de 18 a mais de 58 anos. Constatou-se que mais da metade estava em período produtivo. Estudo realizado com o objetivo de implantar a visita de enfermagem na UTI adulta, verificar e atender as principais necessidades de informação e acolhimento verbalizadas pelas famílias identificou resultado de média de idade dos familiares na faixa dos 40 anos (SIMONI; SILVA, 2012). Desta forma, a família necessita se reorganizar para suprir as atividades antes desenvolvidas pelo familiar internado. Há uma alteração no cotidiano frente à situação de internação de um de seus membros e as tarefas antes exercidas por este acabam por se acumular e necessitam ser assumidas por outro membro da família (WACHTER; BENETTI; BENETTI et al., 2016).

Todos os entrevistados professaram uma religião. Diante de situações em que as pessoas sentemse impotentes frente à resolução de algum problema, acabam por recorrer a algo superior. A religião é utilizada como um instrumento para obter explicações sobre a experiência de doença e morte. A base do comportamento do núcleo familiar frente ao movimento de adaptação à doença e a morte são a religiosidade e a espiritualidade (BOUSSO; POLES; MIRANDA et al., 2011). Este fato evidencia-se nos resultados desta pesquisa.

Múltiplos fatores são responsáveis pelo surgimento e exacerbação do estresse. Os principais estressores foram os relacionados ao paciente, ou seja, ao estado geral e o sofrimento do mesmo. Também evidenciaram que os fatores relacionados à equipe e ao ambiente de UTI foram avaliados como menos estressantes (WACHTER; BENETTI; BENETTI et al., 2016).

O estresse se manifesta por meio de sintomas, fisiológicos e psicológicos, assim, quando há uma resposta ao estresse há uma ativação fisiológica frequente e duradoura a qual pode provocar um esgotamento dos recursos individuais, o que expõe o organismo ao surgimento de diversos transtornos psicofisiológicos os quais podem predispor a doenças (RODRIGUES; SCHIAVO, 2011).
Constata-se nesta pesquisa que todos os participantes apresentaram minimamente um sintoma fisiológico e um psicológico, assim se correlacionou cada fase de estresse para verificar a ocorrência de uma associação significativa entre as mesmas. Em relação ao número de sintomas fisiológicos presentes em cada fase do estresse demonstra-se a existência de uma correlação significativa.

Em uma pesquisa realizada com primigestas usuárias do Sistema Único de Saúde com o objetivo de descrever e comparar as fases do estresse de primigestas no terceiro trimestre de gestação e no pós-parto e correlacioná-las à ocorrência de depressão pós-parto (DPP) houve resultado semelhante. Estes autores detectaram correlação entre os sintomas de estresse apresentados na gestação e os sintomas de depressão pós-parto (RODRIGUES; SCHIAVO, 2011).

Destaca-se que os sintomas psicológicos presentes na fase final do estresse e mencionados pelos pesquisados, apresentaram correlação significativa somente com o total dos referidos sintomas. Quando correlacionado o número de sintomas psicológicos presentes em cada fase, verifica-se que a associação entre a fase inicial, a fase intermediária, a fase final do estresse e o total não são estatisticamente significantes. Este resultado é semelhante ao obtido por em estudo com idosos, o qual objetivou avaliar a frequência de incapacidade funcional associada ao pé doloroso no idoso (PRATO; SANTOS; TREVISANI, 2012).

Verifica-se uma associação estatisticamente significativa quando correlacionado o número de sintomas psicológicos presentes na fase intermediária e com a fase final do estresse e o total. Este dado é semelhante ao obtido em uma pesquisa realizada para avaliar o nível de estresse em mulheres mastectomizadas e examinar a relação do mesmo com variáveis sociodemográficas. Percebeu-se que os sintomas físicos (56\%) predominaram sobre os psicológicos (44\%) (CANICALI; COSTA; SILVEIRA et al., 2013). Com o advento da internação de familiar em UTI ocorrem transformações psicossociais, com alterações na rotina diária, acompanhadas de maior sensibilidade, tristeza, apreensão, medo da perda e dificuldade de conciliar atividades cotidianas às rotinas da UTI (SELL; BETELL; NASCIMENTO et al., 2012). 


\section{CONCLUSÃO}

A internação de um familiar em unidade de tratamento intensivo é um dos principais fatores indutores de estresse, também foi possível evidenciar que as características socioeconômicas dos pesquisados podem, igualmente, contribuir para o estresse. Este por sua vez apresentou-se em níveis elevados, o que é prejudicial ao organismo e contribui para o desencadeamento de doenças.

Nesse contexto, cabe à enfermagem criar estratégias que visem amenizar este sofrimento, as quais iniciam com o acolhimento ao familiar, aliado à comunicação que favoreça o entendimento da real condição do paciente internado. O estresse prolongado, vivenciado pelos pesquisados, além de desencadear inúmeros sintomas psíquicos e fisiológicos, pode contribuir para doenças e, inclusive, a morte prematura.

A dificuldade de encontrar estudos referentes à temática demonstra a relevância de pesquisas, tanto de abordagem quantitativa quanto qualitativa, no sentido de agregar conhecimentos e proporcionar reflexões e ações direcionadas à família do paciente em UTI, e desta forma, qualificar o cuidado de enfermagem.

Os resultados dessa pesquisa podem contribuir com profissionais da saúde, gestores e pesquisadores no sentido de instigá-los a mobilizar ações e intervenções no âmbito hospitalar e na rede básica de saúde, com vistas à uma assistência integral ao familiar que sofre $e$ igualmente necessita ser cuidado.

\section{REFERÊNCIAS}

ABREU, T.G.T.; SENA, L.B.; OLIVEIRA, A.S.; LOPES, M.L.H.; SARDINHA, A.H.L. Cuidadores familiares de idosos portadores de condição crônica. Rev PesqSaúde, v. 14, n.3, p.145-9, 2013.

BAPTISTA, B.O.; BEUTER, M.; PERLINI, N. M. G. O.; BRONDANI, C.M., BUDÓ, M. D.L.D.; SANTOS, N. A sobrecarga do familiar cuidador no âmbito domiciliar: uma revisão integrativa da literatura.Rer.gaúch.enferm, v.33, n.1, p 147-56, 2012.
BARBOSA, M. R.; SANTOS, F. U.; BARBOSA, M. R. Sourcesof stress Patient with diagnosi sofbre ast malignant neoplasia. Rev. bras.ter. cogn., v.8, n.1, p. 10-18, 2012.

BOUSSO, R. S.; POLES, K. S. T. S.; MIRANDA, M. G. Crenças religiosas, doença e morte: perspectiva da família na experiência de doença. Rev. Esc. Enferm. USP, v.45, n.2, p. 397-403, 2011.

CANICALI, P. C.; COSTA, A. M. H.; SILVEIRA, C. D.; CORDEIRO, P. T.; NOGUEIRA, T. P.; MARTINS B. G. B.; COSTA, L. F. M. Estresse em mulheres mastectomizadas. Investigación y Educación en Enfermería, v. 31, n. 3, p. 385-394, 2013.

CAVALCANTE, E. S.; SILVA, R. A. R.; MENDONÇA, A. E. O.; COSTA, M. M. N.; MIRANDA, F.A. N. Evaluation of the stress level of chronic kidney disease patients undergoing hemodialysis treatment. Journal of Nursing UFPE on line, v. 7, n. 5, p. 1264-1270, 2013.

NASCIMENTO, E. R. P., GULINI, J. E. H. M. B., MINUZZI, A. P., RASIA, M.A., DANCZUK, R. F. T., SOUZA, B.C. As relações da enfermagem na unidade de terapia intensiva no olhar de Paterson e Zderad. Revista Enfermagem UERJ, v. 24, n. 2, p. 5817, 2016.

DREFFS, F.M.L.; STUMM, E.M.F.; WINKELMANN, E.R.; UBESSI, L.D. Mecanismos de coping utilizados por familiares de pacientes em terapia intensiva. Revista Ciência \& Saúde, Porto Alegre, v. 6, n. 1, p. 52-58, jan./ abr, 2013.

FERREIRA, P.D.; MENDES, T N.; Família em UTI:: importância do suporte Psicológico diante da iminência de morte. Rev. SBPH, v. 16, n. 1, p. 88-112, 2013.

GOMES, C.M.; CAPELLARI, C.; PEREIRA, D. S.; VOLKART, P.R.; MORAES, A.P.; JARDIM, V. Estresse e risco cardiovascular: intervenção multiprofissional de educação em saúde. Rev. Bras. Enferm, v. 69, n. 2, p. $351-359,2016$

LIPP, M. E. N. Manual do inventário de sintomas de stress para adultos de Lipp (ISSL). São Paulo: Casa do Psicólogo, v. 76, 2000. 
MOMBELLI, M. A.; COSTA, J. B. D.; MOURA, C. .D.; MARCON, S. S. Estrutura e suporte familiar como fatores de risco de stress infantil. Estudos de Psicologia, v. 28, Recebido em: 2017-06-06 n. 3, p. 327-335, 2011.

PRATO, S.C.F.; SANTOS F.C.; TREVISANI, V. F. M. Pé doloroso do idoso associado à incapacidade funcional. Rev. Dor, v. 13, n. 1, p. 18-24, 2012.

RODRIGUES, M .P. R.; SCHIAVO, R. A. Stress na gestação e no puerpério: uma correlação com a depressão pósparto. Rev. Bras. Ginecol.Obstet., v. 33, n. 9, p. 252257, 2011.

SELL, C.T.; BETELL, B.T.; NASCIMENTO, E.R.P.; PADILHA M.I.; CARVALHO J.B. Cuidados intensivos: alterações na dinâmicafamiliar Rev. enferm. UERJ, v. 20, n. 4, p. 48892, 2012.

SIMÕES, J.; BIANCHI, L. R. O. Prevalência da síndrome de Burnout e qualidade do sono em trabalhadores técnicos de enfermagem. Revista Saúde e Pesquisa, v. 9, n. 3, p. 473-481, 2016.

SIMONI, R.C.M.; SILVA, M.J.P. O impacto da visita de enfermagem sobre as necessidades dos familiares de pacientes de UTI. Rev. Esc. enferm. USP, v.46, p. 65-70, 2012.

ZANETTI, T.G.; STUMM, E.M.F.; UBESSI, L. Stress and coping in families of patients in an intensive care unit. Revista de Pesquisa: Cuidado é Fundamental Online, v. 5, n. 2, p. 3608-3619, 2013.

WACHTER, L. G.; BENETTI, P. E.; BENETTI, E. R. R.; CARLI, L. C.; GOMES, J. S.; STUMM, E. M. F. Percebido estresse de membros da família de pacientes renais crônicos em tratamento de hemodiálise. Revista de enfermagem UFPE on-line, v. 10, n. 5, p. 1756-1762, 2016.

WOTTRICH, S. H.; ÁVILA, C. D. M.; MACHADO, C. C.; GOlDMEIER, S.; DILLENBURG, D.; KUHL, C. P.; RUSCHEL, P. P. Gênero e manifestação de stress em hipertensos. Estudos de Psicologia, v. 28, n. 1, p. 27-34, 2011. 